\title{
Received Probability of Orbital-Angular-Momentum Modes Carried by Diffraction- and Attenuation- Resistant Beams in Weak Turbulent Oceans
}

\author{
Yuan Zheng ${ }^{1,+}$, Dongyu Yang ${ }^{1,+}$, Shiqing Qin ${ }^{1}$ and Yixin Zhang ${ }^{2, *}$ \\ 1 School of Science, Jiangnan University, Wuxi 214122, China; r437rt7ds@163.com (Y.Z.); \\ 6181203003@stu.jiangnan.edu.cn (D.Y.); 1132170113@stu.jiangnan.edu.cn (S.Q.) \\ 2 Jiangsu Provincial Research Center of Light Industrial Optoelectronic Engineering and Technology, \\ Wuxi 214122, China \\ * Correspondence: zyx@jiangnan.edu.cn \\ + These authors contributed equally to this work.
}

Received: 25 July 2020; Accepted: 4 September 2020; Published: 10 September 2020

\begin{abstract}
High performance underwater wireless optical communication systems are the key to the construction of high quality underwater optical communication networks. However, seawater absorption and seawater turbulent diffraction should be the main factors limiting the performance of underwater optical communication systems. For this reason, we established the probability model of the orbital angular momentum (OAM) mode received by the communication system to study the influence of the absorbable turbulent seawater channel on the underwater optical communication system with an anti-diffraction and anti-attenuation random (DARR) beam as the signal carrier. In the study, the DARR beam with a large truncated Gaussian aperture was adopted as the signal carrier, seawater absorption was characterized by the complex refractive index of seawater, and seawater turbulence was described by the power spectrum of the refractive index of seawater. By analyzing the relationship between the received probability of the OAM mode of DARR beams and the dissipation rate of kinetic energy per unit of mass of fluid, the ratio of temperature and salinity, dissipation rate of the mean-squared temperature, and other parameters, we show that one can select longer wavelength, smaller OAM quantum number and smaller received diameter to increase the received probability of OAM signal modes. The disturbance of turbulent seawater to the OAM modes with different quantum numbers carried by the DARR beam is less than the corresponding OAM modes carried by the Laguerre-Gaussian beam. Our paper shows that the DARR beam can mitigate the absorption and disturbance of turbulent seawater.
\end{abstract}

Keywords: diffraction-and attenuation-resistant beams; weak turbulent ocean; received probability

\section{Introduction}

Underwater wireless optical communication is crucial to the rapid development of long-range submersible, submarine and marine resources exploration. With the increasing demand for underwater resource development and utilization, the study of underwater optical communication systems with high information capacity has become a research hotspot in the field of underwater optical communication networks [1-6]. Recently, it has become one of the main targets of researchers in this field to improve the theoretical channel capacity of the optical communication system or to control the channel attenuation and channel capacity by selecting a new signal source (beam) [7-17], using adaptive optical systems [18] and by utilizing optimized signal coding technology [19-21]. It is well known that the orbital angular momentum (OAM) mode, carried by photons, provides a new dimension that can be encoded [22], and that the OAM module is related to the spatial distribution of 
wave function [22]. In principle, there is an infinite number of OAM eigenstates available. Therefore the OAM eigenstates (modes) offer the possibilities of realizing arbitrary base-N quantum states and higher capacity optical communication [23], and there is considerable interest in underwater optical communication. However, the spatial nature of the OAM carrier suggests that it may be susceptible to the disturbance of oceanic turbulence [1-17] as well as the absorption of seawater [1,17]. Choosing a special optical signal carrier is one of the effective methods to weaken the turbulent diffraction disturbance or absorption of seawater, so many research results have been reported on the propagation of scalar non-diffraction vortex beams in turbulent seawater, and great progress has been made based on different methods [7-17]. Such progress includes understanding the effect of quasi-nodiffracted beams on the diffraction influence of seawater turbulence. Cheng et al. [7] studied the effect of weak oceanic turbulence and beam source parameters on the propagation of the optical vortexes carried by Bessel-Gaussian (BG) beams based on Rytov approximation, and they attained that BG beams have significant advantages over Laguerre-Gaussian beams in reducing turbulence effects and can improve the performance of OAM-based free-space-optical (FSO) communication links. Later, Zhao et al. [8] analyzed the propagation and self-healing properties of the Bessel-Gaussian vortex beam in an underwater environment. By using the two-frequency coherence function models of a beam in a turbulent medium and the localized wave theory of the polychromatic beam, Wang et al. [9] derived the OAM detection probability of the Airy vortex beam in an anisotropic weak oceanic turbulence and indicated the Airy vortex beam had stronger resistance to oceanic turbulent disturbance when it had a smaller topological charge, larger main ring radius and longer wavelength. Besides, our team has established the crosstalk [10] and the wander [11] models for OAM modes of Lommel-Gaussian beam. We also point out that the Hermi-Gaussian vortex beam [12] and partially coherent Bessel correlation vortex beam [13] can effectively suppress the wavefront distortion caused by turbulent seawater. Further, it was found that Gaussian pulsed X waves and Bessel-Gaussian localized vortex waves $[15,16]$, through turbulent seawater, is higher than that of Laguerre-Gaussian beam in terms of resisting the effects of turbulence. To understand the characteristics of frozen wave resistance diffraction and absorption, Deng et al. [17] established a transmittance model for finite energy frozen beams and analyzed the diffracting- and absorption-resistance of finite energy frozen waves from the beam transmission wavelength, beam waist, beam OAM quantum number, inner scale and outer scale of turbulence, and the ratio of temperature to salinity. By designing longitudinal profile, one can partially compensate the attenuation loss of the transmittance. Due to the advanced manipulation of frozen waves that carry OAM and whose intensity and OAM quantum number can be controlled along the propagation direction, the propagation of waves in free space [24] and in a turbulent atmosphere [25] has attracted much attention recently.Therefore, it is worth discussing whether the anti-absorption and anti-diffraction capability of the underwater wireless optical communication system, which uses the OAM mode carried by the finite energy frozen wave as the signal carrier. However, we have yet to see any reports of research on this issue. In this paper, considering both the turbulence and absorption effects of seawater, we developed a model of received probability of OAM modes carried by a diffraction- and attenuation-resistant beams in turbulent ocean based on the Rytov theory.

In the next section, we briefly analyze the composition of the field of the diffraction- and attenuation-resistant random (DARR) beam (or finite energy random frozen wave) that consists of the frozen wave of the Gaussian apodization. In Section 3, we derive the model for the received probability of the OAM mode when we use DARR beams as the signal carrier in the seawater turbulent communication link. In Section 4, we numerically study the effects of ocean turbulence and beam parameters on the received probability of the OAM signal mode. In Section 5, we give a brief overview and indicate the potential intervals in which DARR might be useful. 


\section{Statistical Description of the Frozen Wave}

Let us consider a diffraction- and attenuation-resistant beam [26] that propagates in a liner, isotropic, and homogeneous absorbing seawater with complex refractive index $n_{\text {sea }}=n_{r}+\mathrm{i} n_{\mathrm{i}}$, where $n_{r}$ and $n_{\mathrm{i}}$ are the real and imaginary parts of the index of refraction of seawater, respectively. As the transmission distance of the light is limited to $200 \mathrm{~m}$ in the "blue-green wavelength range", the diffraction- and attenuation-resistant beam propagates in its Rayleigh range $\left|z_{R}\right|=$ $w_{0}^{2} \omega \sqrt[2]{n_{r}^{2}+n_{i}^{2} / 2 c}$, in which, $\omega$ is the angular frequency, $c$ is the speed of light in a vacuum and $w_{0}$ is the width of light beam.

We know that the wavefront of a Gaussian apodization located within its Rayleigh range $\left|z_{R}\right|$ is similar to the wavefront of a plane wave [27]. Considering that the diffraction- and attenuation-resistant beam is composed of a weighted superposition of Bessel light waves and Bessel light wave is composed of regular plane wave superposition. Hence, if the apodization width $w_{0}$ of the Gaussian truncated diffraction-and attenuation-resistant beam is much larger than the received aperture, the Gaussian truncated effect of the beam can be ignored. In the following research, we use Equation (1) to describe Gaussian truncated diffraction- and attenuation-resistant beam.

In cylindrical coordinates $(r, \varphi, z)$, the complex amplitude of a diffracting- and absorption-resistance beam with large apodization is approximately by a superposition of $2 \mathrm{~N}+1$ co-propagation first kind Bessel beams of order $l_{0}$ and angular frequency $\omega$ [28]:

$$
E_{0}(r, \varphi, z, t) \approx N_{l 0} \exp (-\mathrm{i} \omega t) \exp \left(\mathrm{i} l_{0} \varphi\right) \sum_{n=-N}^{N} A_{n} J_{l 0}\left(\eta_{n} r\right) \exp \left(\mathrm{i} \zeta_{n} z\right)
$$

Here, apodization width $w_{0}>D / 2, \mathrm{D}$ is the diameter of the receiver aperture. $z$ is the position of any point along the optical axis, $N_{l 0}=1 /\left[J_{l 0}(\cdot)\right]_{\max },\left[J_{l 0}(\cdot)\right]_{\max }$ is the maximum value of the first kind Bessel function of order $l_{0}, A_{n}$ is a superimposed weight coefficient, $\zeta_{n}$ and $\eta_{n}$ are the longitudinal and transverse wavenumbers of the nth Bessel beam; $\zeta_{n}=Q+2 \pi n / L+\mathrm{i}(Q+2 \pi n / L) n_{\mathrm{i}} / n_{r}$, $Q>0$ is a value to be chosen according to the given experimental situation, and the desired degree of transverse field localization [26,28]; $L$ is the propagation length of the beam. $\eta_{n}=$ $\left[\left(\left(n_{r}^{2}-n_{\mathrm{i}}^{2}\right) \frac{4 \pi^{2}}{\lambda^{2}}-\left(Q+\frac{2 \pi n}{L}\right)^{2}+\left(\frac{4 \pi^{2}}{\lambda^{2}} \frac{n_{r} n_{\mathrm{i}}}{Q+2 \pi n / L}\right)^{2}\right]^{1 / 2}\right.$, and $\lambda$ is the vacuum wavelength of light waves.

When a diffracting- and absorption-resistance beam propagates in absorbable turbulent seawater, turbulence produces an additional phase $\psi(r, \varphi, z)$ in the wave-front of the beam, and the $\psi(r, \varphi, z)$ is a zero mean Gaussian distribution random function [29]. For weak turbulent fluctuations, the field amplitude of transmitted optical signals can be approximately calculated by Rytov approximation. In mathematics, the Rytov approximation relates the field transmitted in a turbulent medium to the field transmitted in a medium without turbulence using the following formula

$$
E(r, \varphi, z, t)=E_{0}(r, \varphi, z, t) \exp [\psi(r, \phi, z)]
$$

The field represented by Equation (2) is the field of the DARR beam—namely [17,29]

$$
E(r, \varphi, z, t)=N_{l 0} \exp (-\mathrm{i} \omega t) \exp \left(\mathrm{i} l_{0} \varphi+\psi(r, \phi, z)\right) \sum_{n=-N}^{N} A_{n} J_{l 0}\left(\eta_{n} r\right) \exp \left(\mathrm{i} \zeta_{n} z\right)
$$

In frozen wave theory $[26,28]$, to obtain the longitudinal intensity profile, set $\left|E\left(r_{0}, \varphi, z\right)\right|^{2} \approx|F(z)|^{2}$, within $0 \leq z \leq L[26,28]$. Here $F(z)$ is the longitudinal intensity profile function, $r_{0}$ is the parameter related to the zero position of the first kind of Bessel function. This paper, however, studies the transmission of the DARR beam in turbulent seawater, the method proposed in frozen wave theory $[26,28]$ to determine the superposition coefficients of the diffracting- and absorption-resistance beam in homogeneous medium, which are no longer applicable. Therefore, we must use stochastic 
statistical theory to determine the superposition coefficients. Utilizing the zero mean Gaussian random field statistical method, we can determine the light intensity of the DARR beam on a cylindrical surface of radius $r=r_{0}$ for $0 \leq z \leq L$ in the following relation [17]

$$
\left.\left.\langle| E\left(r=r_{0}, \varphi, z\right)\right)\left.\right|^{2}\right\rangle \approx|F(z)|^{2},
$$

Note that the symbol $<\cdot>$ means the statistical average of the turbulence ensemble. Substituting Equation (3) into Equation (4), we get the following statistical average relation:

$$
\begin{aligned}
\left\langle\left|E\left(r=r_{0}, \varphi, z\right)\right|^{2}\right\rangle= & \mid N_{l 0}\left\langle\exp \left[\psi r, \varphi, z+\psi^{*} r, \varphi, z\right]\right\rangle^{1 / 2} \\
& \times\left.\sum_{n=-N}^{N} A_{n} J_{l 0}\left(\eta_{n} r\right) \exp (\mathrm{i} 2 \pi n z / L)\right|^{2},
\end{aligned}
$$

in here, the term $\left\langle\exp \left(\Psi(r, \varphi, z)+\Psi^{*}(r, \varphi, z)\right\rangle\right.$ satisfies following expression [29]

$$
\begin{aligned}
\left\langle\exp \left(\Psi(r, \varphi, z)+\Psi^{*}(r, \varphi, z)\right\rangle\right. & =\exp \left(-r^{2} / \rho_{o c}^{2}\right) \\
& =\exp \left(-r^{2} \rho_{\xi}^{2} z\right),
\end{aligned}
$$

where $\rho_{o c}=\left(\rho_{\xi}^{2} z\right)^{-1 / 2} \approx\left[\pi^{2}|k|^{2} z \int_{0}^{\infty} \kappa^{3} \Phi_{n}(\kappa) d \kappa\right]^{-1 / 2}$ is the spatial coherence radius of a plane wave propagating in the oceanic turbulence, $k=2 \pi n_{\text {sea }} / \lambda, \kappa$ is the spatial frequency of the refractive index fluctuations, and $\Phi_{n}$ is the power spectrum of refractive-index fluctuations.

From Equation (6), we can express the relationship $\left\langle\exp \left[\psi(r, \varphi, z)+\psi^{*}(r, \varphi, z)\right]\right\rangle^{1 / 2}$ in Equation (3) as

$$
\left\langle\exp \left[\psi(r, \varphi, z)+\psi^{*}(r, \varphi, z)\right]\right\rangle^{1 / 2}=\exp \left(-r^{2} \rho_{\xi}^{2} z / 2\right) .
$$

To simplify the subsequent analysis, in this paper, we use the power spectrum of refractive-index fluctuations for $C_{0}=0.72$ and $A=2.6 \times 10^{-4}$ liter $/ \operatorname{deg}[11]$ - that is

$$
\begin{gathered}
\Phi_{n}(\kappa)=\frac{4.9 \times 10^{-8} \chi_{T}\left[1+C_{1}(\kappa \eta)^{2 / 3}\right]}{4 \pi \varepsilon^{1 / 3} \omega^{2}\left(\kappa^{2}+\kappa_{0}^{2}\right)^{11 / 6}}\left[\mathcal{D}^{2} e^{-\kappa^{2} \eta^{2} / R_{T}^{2}}\right. \\
\left.+\frac{e^{-\kappa^{2} \eta^{2} / R_{S}^{2}}}{d_{r}}-\left(1+\frac{1}{d_{r}}\right) \omega e^{-\kappa^{2} \eta^{2} / R_{T S}^{2}}\right], 0<\kappa<\infty
\end{gathered}
$$

and

$$
d_{r}=\frac{|\omega|}{R_{F}} \approx \begin{cases}1 /(1-\sqrt{(1-(1 /|\omega|)}), & |\omega| \geq 1 \\ 1.85|\omega|-0.85, & 0.5 \leq|\omega| \leq 1 \\ 0.15 \mid \omega, & |\omega| \leq 0.5\end{cases}
$$

where $\omega$ defines the ratio of temperature and salinity contributions to the refractive index fluctuations, which can vary in the interval $[-5,0]$ in the seawater, with -5 and 0 corresponding to dominating temperature-induced and salinity-induced optical turbulence [30], respectively. $\varepsilon$ is the rate of dissipation of kinetic energy per unit mass of fluid ranging from $10^{-10} \mathrm{~m}^{2} / \mathrm{s}^{3}$ to $10^{-1} \mathrm{~m}^{2} / \mathrm{s}^{3} ; \chi_{T}$ is the dissipation rate of the mean-squared temperature and has the range from $10^{-10} \mathrm{~K}^{2} / \mathrm{s}$ to $10^{-4} \mathrm{~K}^{2} / \mathrm{s} ; R_{F}$ is the eddy flux ratio, $\kappa_{0}=2 \pi / L_{0}, L_{0}$ is the outer scale of turbulence, $C_{1}$ is a free parameter, $\eta$ is the inner scale, $R_{T, S, T S}=\sqrt{3}\left[W_{T, S, T S}-1 / 3+1 /\left(9 W_{T, S, T S}\right)\right]^{3 / 2} / q^{3 / 2}, W_{T, S, T S}=$ $\left\{\left[\operatorname{Pr}_{T, S, T S}^{2}\left(6 \gamma q^{-2}\right)^{2}-\operatorname{Pr}_{T, S, T S} / 81 \gamma q^{-2}\right]^{1 / 2}-\left[1 / 27-\operatorname{Pr}_{T, S, T S} /\left(6 \gamma q^{-2}\right]\right\}^{1 / 3}, \operatorname{Pr}_{T}, \operatorname{Pr}_{S}\right.$ represent the Prandtl numbers of the temperature and salinity, respectively, and $P r_{T S}=2 P r_{T} P r_{S} /\left(P r_{T}+P r_{S}\right)$, $q$ is the non-dimensional constant. 
Consider the integral expression of the confluent hypergeometric function of the second kind $\mathrm{U}(a ; b ; c)[31]$ :

$$
\begin{gathered}
\mathrm{U}\left(\mu+1 / 2 ; \mu-1 / 3 ; \kappa_{0}^{2} / \kappa_{H}^{2}\right) \\
=\frac{2}{\kappa_{0}^{2 \mu-8 / 3} \Gamma(\mu+1 / 2)} \int_{0}^{\infty} \frac{\kappa^{2 \mu}}{\left(\kappa^{2}+\kappa_{0}^{2}\right)^{-11 / 6}} e^{-\kappa^{2} / \kappa_{H}^{2}} d \kappa .
\end{gathered}
$$

We expressed the coherence length of the plane wave as [11,17]

$$
\begin{gathered}
\rho_{o c}=\left(\frac { 0 . 7 5 8 \times 1 0 ^ { - 6 } } { \lambda ^ { 2 } } \varepsilon ^ { - 1 / 3 } \chi _ { T } ( { \overline { n _ { r } } } ^ { 2 } + { \overline { n _ { \mathrm { i } } } } ^ { 2 } ) \pi z \left\{\kappa_{0}^{1 / 3}\right.\right. \\
\times\left[\mathrm{U}\left(2 ; \frac{7}{6} ; \frac{\kappa_{0}^{2} \eta_{2}}{R_{T}^{2}}\right)+\frac{1}{d_{r} \omega^{2}} \mathrm{U}\left(2 ; \frac{7}{6} ; \frac{\kappa_{0}^{2} \eta_{2}}{R_{S}^{2}}\right)-\frac{2\left(1+d_{r}\right)}{\omega d_{r}}\right. \\
\left.\times \mathrm{U}\left(2 ; \frac{7}{6} ; \frac{\kappa_{0}^{2} \eta_{2}}{R_{T S}^{2}}\right)\right]+C_{1} \eta^{2 / 3} \kappa_{0} \Gamma\left(\frac{7}{3}\right)\left[\mathrm{U}\left(\frac{7}{3} ; \frac{3}{2} ; \frac{\kappa_{0}^{2} \eta_{2}}{R_{T}^{2}}\right)\right. \\
\left.\left.\left.+\frac{1}{d_{r} \omega^{2}} \mathrm{U}\left(\frac{7}{3} ; \frac{3}{2} ; \frac{\kappa_{0}^{2} \eta_{2}}{R_{S}^{2}}\right)-\frac{2\left(1+d_{r}\right)}{\omega d_{r}} \mathrm{U}\left(\frac{7}{3} ; \frac{3}{2} ; \frac{\kappa_{0}^{2} \eta_{2}}{R_{T S}^{2}}\right)\right]\right\}\right)^{-\frac{1}{2}},
\end{gathered}
$$

where $\Gamma(\cdot)$ is a gamma function.

From the previous discussion, the longitudinal intensity profile function $F(z)$ can be expanded, as in $[26,28]$, in a Fourier series

$$
F(z) \approx \mathrm{e}^{-r_{0}^{2} \rho_{\xi}^{2} z / 2} \sum_{n=-N}^{N} A_{n} e^{\mathrm{i} 2 \pi n z / L},
$$

where the Fourier coefficient $A_{n}$ is given by

$$
A_{n}=\frac{1}{L} \int_{0}^{L} F(z) \mathrm{e}^{\left(-r_{0}^{2} \rho_{\xi}^{2} z / 2-\mathrm{i} 2 \pi n z / L\right) z} d z
$$

If we consider the longitudinal intensity profile function

$$
F(z)= \begin{cases}0, & 0<z<40 \mathrm{~m} \\ 1, & 40 \leq z \leq 50 \mathrm{~m} \\ 0, & 50<z<90 \mathrm{~m} \\ 1, & 90 \leq z \leq 100 \mathrm{~m} \\ 0, & 100<z<140 \mathrm{~m} \\ 1, & 140 \leq z \leq 150 \mathrm{~m}\end{cases}
$$

we have a simplification relationship

$$
A_{n}= \begin{cases}\frac{\mathrm{e}^{\left(\frac{r_{0}^{2} \rho_{\xi}^{2}}{2}-\frac{\mathrm{i} 2 \pi n}{L}\right) z_{1}}-\mathrm{e}^{\left(\frac{r_{0}^{2} \rho_{\xi}^{2}}{2}-\frac{\mathrm{i} 2 \pi n}{L}\right) z_{2}}}{L\left(r_{0}^{2} \rho_{\xi}^{2} / 2-\mathrm{i} 2 \pi n / L\right)}, & F(z)=1 \\ 0, & F(z)=0\end{cases}
$$

where $z_{1}$ represents the starting position of transverse profile monitoring (extraction), and $z_{2}$ is the termination position of the transverse profile monitoring.

\section{Received Probability of Orbital-Angular-Momentum Modes}

Based on the analysis in the above section, we now turn to the establishment of the Received probability model of OAM modes. As the beam propagates through the oceanic turbulence, 
the eigenstate of OAM carried by the DARR beam is no longer guaranteed to be in the original eigenstate of OAM. However, by the superposition theory of waves, the disturbed field of the DARR beam can be decomposed into a series of spiral harmonics $e^{\mathrm{i} l \varphi}$ with expansion coefficient $B_{l}$ as follows $[10,17]$

$$
E(r, \varphi, z, t)=\frac{1}{\sqrt{2 \pi}} \sum_{l=-\infty}^{\infty} B_{l} \exp (\mathrm{i} l \varphi)
$$

The ensemble average of $\left|B_{l}\right|^{2}$ represents the probability density of vortex mode in oceanic turbulence, which has the form of

$$
\begin{gathered}
\left\langle\left|B_{l}^{2}\right|\right\rangle \quad=\left|\int_{2}^{2 \pi} \int_{2}^{2 \pi} E(r, \varphi, z, t) E^{*}\left(r, \varphi^{\prime}, z, t\right) \frac{e^{-\mathrm{i} l\left(\varphi-\varphi^{\prime}\right)}}{2 \pi} d \varphi d \varphi^{\prime}\right| \\
=\frac{N_{l 0}^{2}}{2 \pi}\left|\sum_{n=-N}^{N} A_{n} J_{l 0}\left(\eta_{n} r\right) e^{\mathrm{i} 2 \pi n z / L}\right|^{2} e^{-2 r^{2} / \rho_{o c}^{2}} \\
\times\left|\int_{0}^{2 \pi}\left[\int_{0}^{2 \pi} e^{-\mathrm{i}\left(l-l_{0}\right) \varphi} e^{2 r^{2} \cos \left(\varphi^{\prime}-\varphi\right) / \rho_{o c}^{2}}\right] e^{-\mathrm{i}\left(l-l_{0}\right) \varphi^{\prime}}\right| d \varphi d \varphi^{\prime} .
\end{gathered}
$$

In the above equation, we have already used the relationship: $\left\langle e^{\psi(r, \varphi, z)+\psi^{*}(r, \varphi, z)}\right\rangle=$ $e^{-2 r^{2}\left[1-\cos \left(\varphi-\varphi^{\prime}\right)\right] / \rho_{o c}^{2}[17] \text {. }}$

Consider the expression of modified Bessel function of imaginary argument $I_{m}(x)$ [7]

$$
\int_{0}^{2 \pi} e^{x \cos \left(\varphi^{\prime}-\varphi\right)-\mathrm{i} m \varphi d \varphi^{\prime}=2 \pi e^{-\mathrm{i} m \varphi} I_{m}(x)}
$$

Equation (17) is simplified to

$$
\begin{aligned}
\left\langle\left|B_{l}\right|^{2}\right\rangle & =N_{l 0}^{2} \exp \left(-\frac{2 r^{2}}{\rho_{o c}^{2}}\right)\left|I_{l-l_{0}}\left(\frac{2 r^{2}}{\rho_{c c}^{2}}\right)\right| \\
& \times\left|\sum_{n=-N}^{N} A_{n} J_{l 0}\left(\eta_{n} r\right) e^{\mathrm{i} 2 \pi n z / L}\right|^{2} .
\end{aligned}
$$

In seawater communication, we focus on signal transmission. According to Equation (19), we can get the probability density of OAM signal modes for $l-l_{0}=0$ and $I_{l-l_{0}}\left(2 r^{2} / \rho_{o c}^{2}\right)=I_{0}\left(2 r^{2} / \rho_{o c}^{2}\right)$

$$
\begin{aligned}
&\left\langle\left|B_{l}\right|^{2}\right\rangle=N_{l 0}^{2} \exp \left(-\frac{2 r^{2}}{\rho_{o c}^{2}}\right)\left|I_{0}\left(\frac{2 r^{2}}{\rho_{c c}^{2}}\right)\right| \\
& \times\left|\sum_{n=-N}^{N} A_{n} J_{l 0}\left(\eta_{n} r\right) e^{\mathrm{i} 2 \pi n z / L}\right|^{2} .
\end{aligned}
$$

The received probability of OAM signal modes of the DARR beam is defined as

$$
P\left(l_{0}\right)=\frac{\int_{0}^{D / 2} e^{-\frac{2 r^{2}}{\rho_{o c}}}\left|I_{0}\left(\frac{2 r^{2}}{\rho_{c c}^{2}}\right)\right|\left|\sum_{n=-N}^{N} A_{n} J_{l 0}\left(\eta_{n} r\right) e^{\mathrm{i} 2 \pi n z / L}\right|^{2} r d r}{\sum_{l=-\infty}^{\infty} \int_{0}^{D / 2} e^{-\frac{2 r^{2}}{\rho_{c c}}}\left|I_{0}\left(\frac{2 r^{2}}{\rho_{o c}^{2}}\right)\right|\left|\sum_{n=-N}^{N} A_{n} J_{l 0}\left(\eta_{n} r\right) e^{\mathrm{i} 2 \pi n z / L}\right|^{2} r d r} .
$$

The decrease in the received probability of the signal OAM mode indicates the loss of the emitting signal OAM mode caused by oceanic turbulence.

\section{Numerical Results and Analysis}

In this section, we numerically analyzed the evolution of DARR beams in the oceanic turbulence with the parameter variation of beam and seawater channel in the seawater "transmission window" region. In numerical calculation, unless otherwise stated, the parameters are set as: $\lambda=410 \mathrm{~nm}$, $E_{0}=1, L=150 \mathrm{~m}$ (in Rayleigh distance), $l_{0}=1, r_{0}=8.1795 \times 10^{-5} \mathrm{~m}, w_{0}=2.5 \times 10^{3} r_{0}, \varepsilon=1 \times 10^{-6}$ 
$\mathrm{m}^{2} / \mathrm{s}^{3}, q=2.5, \chi_{T}=1 \times 10^{-8} \mathrm{~K}^{2} / \mathrm{s}, L_{0}=10 \mathrm{~m}, P_{r s}=700, D=0.15 \mathrm{~m}, n_{o c}=1.34+1.0114 \times 10^{-10} \mathrm{i}$, $\mathscr{\omega}=-1, \eta=1 \mathrm{~mm}, N=15, P_{r t}=7, F(z)=1$ and $C_{1}=2.35$. Considering the value $Q$ must be chosen within the interval $\left[0 \leq Q \pm \frac{2 \pi}{L} N \leq \frac{2 \pi}{\lambda}\right]$, we chose $Q=0.9999994 \mathrm{k}$.

Figure 1 plots the received probability of the DARR beam versus propagation distance $z$ for different OAM signal modes $l_{0}$. Figure 1 shows that the probability decreases as propagate distance $z$ increases. Nevertheless, it shows that the received probability is still over 0.8 in the receiving plane. In any case, Figure 1 reveals that the OAM mode of the DARR beam with lower $l_{0}$ is affected lower by the absorbable turbulent seawater. However, the effect of absorbable turbulent seawater on the OAM signal modes of different orders is very small. The results show that the absorbable turbulent seawater makes little difference in the disturbance of vortex modes of different orders carried by the frozen waves. The result is different from the Laguerre-Gaussian beam [2].

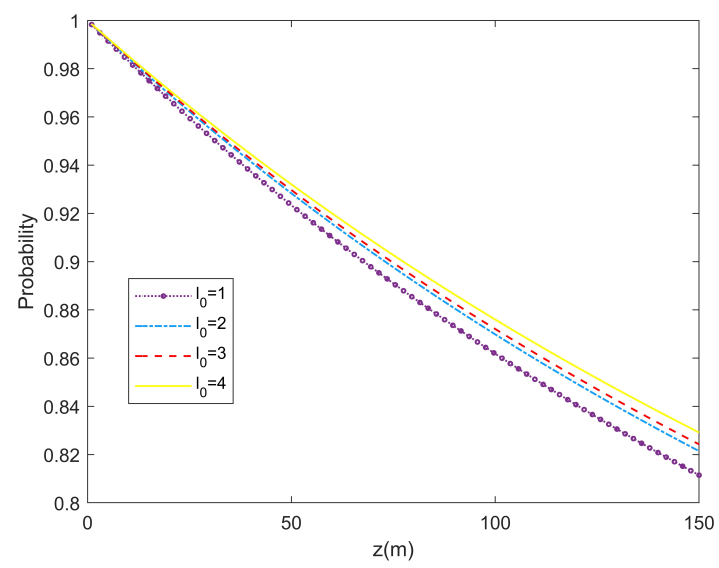

Figure 1. Received probability of the OAM signal modes of DARR beam versus propagate distance $\mathrm{Z}$ for different OAM mode $l_{0}$.

In Figure 2, we investigate the relationship between the received probability of the DARR beam and the temperature-salinity contribution ratios $\omega$. The numerical curves in the figure show that, with the increase in $\omega$, the received probability goes down-especially when $\omega$ is within the interval $[-1,0]$, the received probability decreases rapidly. This result indicates that, similar to other beams [7-17], the OAM modes of the DARR beam are more affected by the fluctuation in seawater salinity than by the fluctuation in seawater temperature.

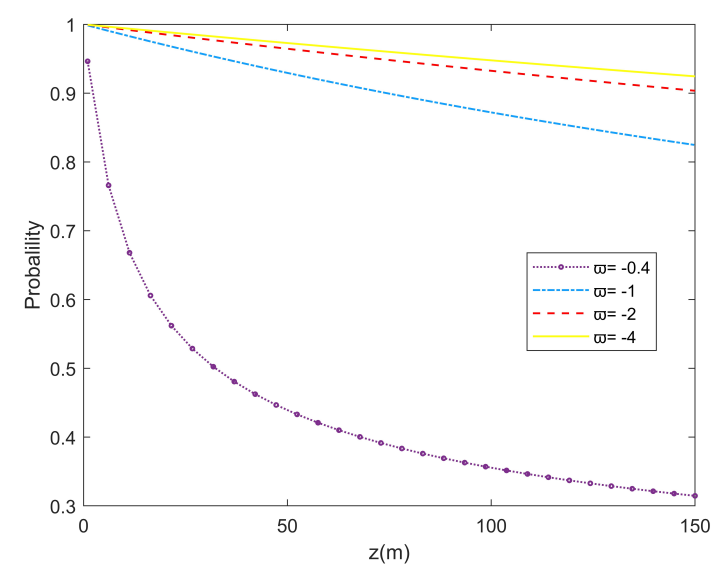

Figure 2. Received probability of the OAM signal modes of DARR beam versus propagate distance $\mathrm{Z}$ for different temperature-salinity contribution ratios $\omega$. 
Figure 3 displays the effect of dissipation rates of the mean-squared temperature and of kinetic energy per unit mass of fluid on the received probability of the OAM signal modes of the DARR beam. The surface in Figure 3 shows that the lower $\chi_{T}$ and the higher $\varepsilon$ will have higher received probability of the OAM signal mode of the DARR beam. The phenomenon can be explained through lower dissipation rates of the mean-squared temperature and a higher rate of dissipation of kinetic energy per unit mass of fluid, which corresponds to lower oceanic turbulence [7-17].

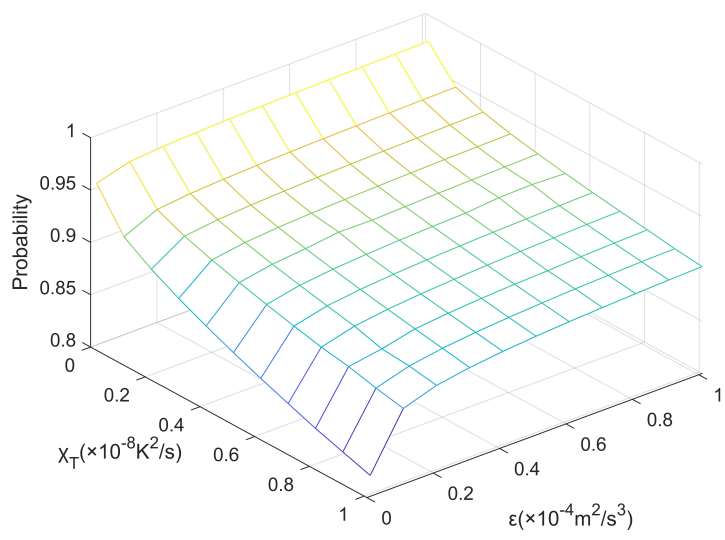

Figure 3. Received probability of the OAM signal modes of DARR beam versus dissipation rates of the mean-squared temperature $\chi_{T}$ and the dissipation rates of kinetic energy per unit mass of fluid $\varepsilon$.

We also analyzed the impact of inner and outer scales of turbulence on the received probability of the OAM mode of the DARR beam. Figure 4 shows that the received probability of the OAM mode increases with the increase in the inner and decrease in the outer scales. This result mainly comes from the fact that the larger turbulent inner scale corresponds to the smaller light scattering, which leads to the lesser energy scattering loss of the OAM mode. Similarly, the smaller turbulent outer scale corresponds to the smaller beam drift, which leads to the smaller deflection energy loss of the OAM mode [32].

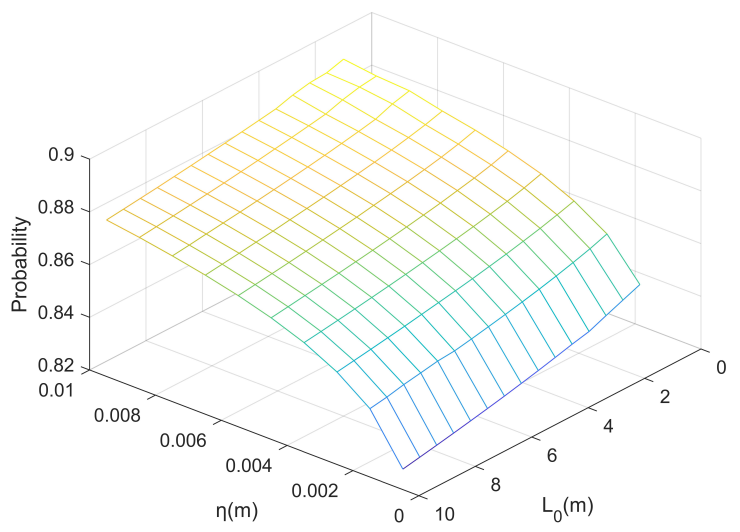

Figure 4. Received probability of the OAM signal modes of DARR beam versus inner scale $\eta$ and outer scale $L_{0}$.

In Figure 5, we investigate the influence of received diameter $D$ and the wavelength $\lambda$ of the DARR beam on the received probability of the OAM mode. Figure 5 shows that the received probability becomes greater as the wavelength of the beam increases. The result is that the turbulent scintillation of the long-wave beam is smaller than that of the short wavelength beam [29] —-this suggests that the beam is less disturbed by turbulence, so the OAM mode with long-wavelength has higher received 
probability. In addition, it is depicted in the figure that the received probability decreases with the increase in the receiver aperture, and finally approaches a constant. This conclusion is consistent with the results obtained for the study of pure vortex beam propagation in a turbulent atmosphere [33]. That is, in the cases of very small collecting apertures, the received probability of the OAM signal mode decreases with the increase in the aperture size, and, in the cases of very large collecting apertures, the received probability of the OAM crosstalk mode tends towards zero. The main reason for this phenomenon is that, when the signal is received with a large receiving aperture, the phase difference between the incident rays to the receiver is large, resulting in the superposition noise generated when the beams are coherently superpositioned. Of course, in this case, the probability of the received OAM signal mode is small. Finally, when the receiving aperture is large enough that the receiving spot falls completely within the receiving aperture, then when the receiving aperture increases further, the receiving probability of OAM mode will not change.

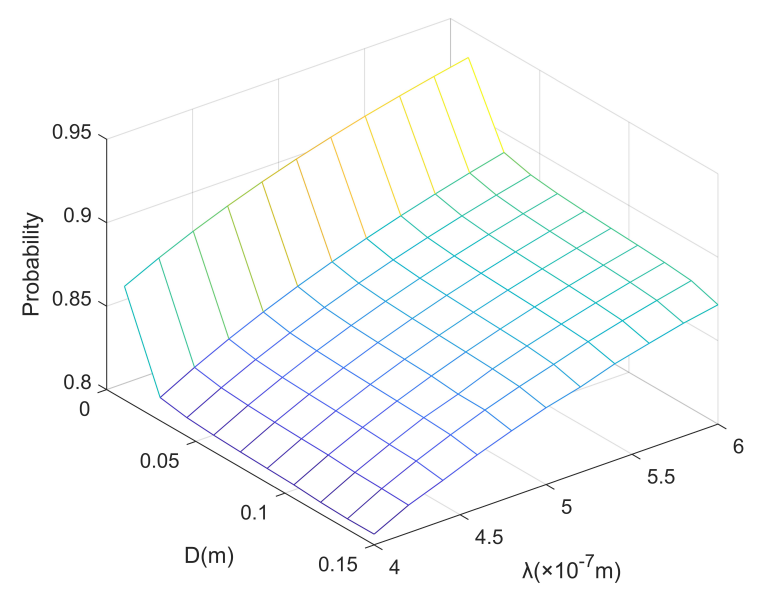

Figure 5. Received probability of the OAM signal modes of DARR beam versus wavelength $\lambda$ and received diameter $\mathrm{D}$.

\section{Conclusions}

In this paper, using Rytov and the frozen wave theory, we developed the received probability of the OAM mode of the DARR beam in seawater with weak turbulence and absorption. By this model, we reached the following significant conclusions: The OAM modes of the DARR beam are more affected by the fluctuation in seawater salinity than by the fluctuation in seawater temperature, and the OAM mode of the DARR beam with lower $l_{0}$ and longer wavelength is less affected by the turbulence and absorption of seawater. As well as this, sub-sea communication systems with smaller signal receiving diameters are less disturbed by seawater, and the OAM mode carried by the DARR beam has good characteristics of diffraction- and attenuation-resistance, even in long-distance seawater turbulence channels. However, it should be noted that, as the receiver aperture of the communication system is close to the transmitter aperture, it is necessary to replace the approximate model (1) with an accurate finite energy frozen wave model [28].

Author Contributions: Y.Z. (Yuan Zheng): figures and writing; D.Y.: literature search and data analysis; S.Q.: data collection; Y.Z. (Yixin Zhang): study design and data interpreation. All authors have read and agreed to the published version of the manuscript.

Funding: This research was funded by the National Natural Science Foundation of China (NSFC) (Grant No. 61871202) and National Natural Science Foundation of China (NSFC) (Grant No. 61701196).

Acknowledgments: This work was supported by project of Innovation and Entrepreneurship Training Program of Jiangnan University (Item Number: 2019374Y), and the Postgraduate Research Practice Innovation Program of Jiangsu province (SJCX20_0764).

Conflicts of Interest: The authors declare no conflict of interest. 


\section{References}

1. Zeng, Z.; Fu, S.; Zhang, H.; Dong, Y.; Cheng, J. A survey of underwater optical wireless communications. IEEE Commun. Surv. Tutor. 2017, 19, 204-238. [CrossRef]

2. Zhang, W.; Wang, L.; Wang, W.; Zhao, S. Propagation property of Laguerre-Gaussian beams carrying fractional orbital angular momentum in an underwater channel. OSA Continuum. 2019, 2, 3281-3297. [CrossRef]

3. Xia, T.; Liu, D.; Dong, A.; Wang, G.; Zhong, H.; Wang, Y. Properties of partially coherent elegant Laguerre-Gaussian beam in free space and oceanic turbulence. Optik 2020, 201, 163514. [CrossRef]

4. Cheng, M.; Guo, L.; Li, J.; Huang, Q.; Cheng, Q.; Zhang, D. Propagation of an optical vortex carried by a partially coherent Laguerre-Gaussian beam in turbulent ocean. Appl. Opt. 2016, 55, 4642-4648. [CrossRef] [PubMed]

5. Li, Y.; Zhang, Y.; Zhu, Y. Probability distribution of the orbital angular momentum mode of the ultrashort Laguerre-Gaussian pulsed beam propagation in oceanic turbulence. Results Phys. 2018, 11, 698-705. [CrossRef]

6. Pan, S.; Wang, L.; Wang, W.; Zhao, S. An effective way for simulating oceanic turbulence channel on the beam carrying orbital angular momentum. Sci. Rep. 2019, 9, 14009. [CrossRef]

7. Cheng, S.M.; Guo, L.; Li, J.; Zhang, Y. Channel capacity of the OAM-based free-space optical communication Links with Bessel-Gauss beams in turbulent ocean. IEEE Photon. J. 2016, 8, 7901411. [CrossRef]

8. Zhao, S.; Zhang, W.; Wang, L.; Li, W.; Gong, L.; Cheng, W.; Chen, H.; Gruska, J. Propagation and self-healing properties of Bessel-Gaussian beam carrying orbital angular momentum in an underwater environment. Sci. Rep. 2019, 9, 2025. [CrossRef]

9. Wang, X.; Yang, Z.; Zhao, S. Influence of oceanic turbulence on propagation of Airy vortex beam carrying orbital angular momentum. Optik 2019, 176, 49-55. [CrossRef]

10. Yu, L.; Zhang, Y. Analysis of modal crosstalk for communication in turbulent ocean using Lommel-Gaussian beam. Opt. Express 2017, 25, 22565-22574. [CrossRef]

11. Liang, Q.; Zhu, Y.; Zhang, Y. Approximations wander model for the Lommel Gaussian-Schell beam through unstable stratification and weak ocean-turbulence. Results Phys. 2019, 14, 102511. [CrossRef]

12. Li, Y.; Yu, L.; Zhang, Y. Influence of anisotropic turbulence on the orbital angular momentum modes of Hermite-Gaussian vortex beam in the ocean. Opt. Express 2017, 25, 12203-12215. [CrossRef] [PubMed]

13. Xu, Y.; Shi, H.; Zhang, Y. Effects of anisotropic oceanic turbulence on the power of the bandwidth-limited OAM mode of partially coherent modified Bessel correlated vortex beams. J. Opt. Soc. Am. A 2018, 35, 1839-1845. [CrossRef] [PubMed]

14. Li, Y.; Zhang, Y.; Zhu, Y. Influence of oceanic turbulence on propagation of the Gaussian pulsed X wave carrying orbital angular momentum. Opt. Commun. 2018, 428, 57-62. [CrossRef]

15. Yang, D.; Zhang, Y.; Shi, H. Capacity of turbulent ocean links with carrier Bessel-Gaussian localized vortex waves. Appl. Opt. 2019, 58, 9484-9490. [CrossRef]

16. Deng, S.; Zhu, Y.; Zhang, Y. Received probability of vortex modes carried by localized wave of Bessel-Gaussian amplitude envelope in turbulent seawater. J. Mar. Sci. Eng. 2019, 7, 203. [CrossRef]

17. Deng, S.; Yang, D.; Zheng, Y.; Hu, L.; Zhang, Y. Transmittance of finite-energy frozen beams in oceanic turbulence. Results Phys. 2019, 15, 102802. [CrossRef]

18. Yin, X.; Chang, H.; Cui, X.; Ma, J.; Wang, Y.; Wu, G.; Zhang, L.; Xin, X. Adaptive turbulence compensation with a hybrid input-output algorithm in orbital angular momentum-based free-space optical communication. Appl. Opt. 2018, 57, 7644-7650. [CrossRef]

19. Cui, X.; Yin, X.; Chang, H.; Liao, H.; Chen, X.; Xin, X.; Wang, Y. Experimental study of machine-learning-based orbital angular momentum shift keying decoders in optical underwater channels. Opt. Commun. 2019, 452, 116-123. [CrossRef]

20. Cui, X.; Yin, X.; Chang, H.; Guo, Y.; Zheng, Z.; Sun, Z.; Liu, G.; Wang, Y. Analysis of an adaptive orbital angular momentum shift keying decoder based on machine learning under oceanic turbulence channels. Opt. Commun. 2018, 429, 138-143. [CrossRef]

21. Wang, W.; Wang, P.; Cao, T.; Tian, H.; Zhang, Y.; Guo, L. Performance investigation of underwater wireless optical communication system using M-ary OAMSK modulation over oceanic turbulence. IEEE Photon. J. 2017, 9, 7905315. [CrossRef] 
22. Allen, L.; Beijersbergen, M.W.; Spreeuw, R.J.C.; Woerdman, J.P. Orbital angular momentum of light and the transformation of Laguerre-Gaussian laser modes. Phys. Rev. A 1992, 45, 8185. [CrossRef] [PubMed]

23. Mair, A.; Vaziri, A.; Weihs, G.; Zeilinger, A. Entanglement of the orbital angular momentum states of photons. Nature 2001, 412, 313-316. [CrossRef] [PubMed]

24. Dorrah, A.H.; Zamboni-Rached, M.; Mojahedi, M. Controlling the topological charge of twisted light beams with propagation. Phys. Rev. A 2016, 93, 063864. [CrossRef]

25. Tian, Q.; Zhu, L.; Wang, Y.; Zhang, Q.; Liu, B.; Xin, X. The Propagation Properties of a Longitudinal Orbital Angular Momentum Multiplexing System in Atmospheric Turbulence. IEEE Photonics J. 2018, 10, 1-16. [CrossRef]

26. Zamboni-Rached, M.; Ambrósio, L.A.; Hernández-Figueroa, H.E. Diffraction-attenuation resistant beams: Their higher-order versions and finite-aperture generations. Appl. Opt. 2010, 49, 5861-5869. [CrossRef]

27. Davis, C. DLaser and Electro-Optics; McGraw-Hill: Cambridge, UK; New York, NY, USA, 1996; Volume 49.

28. Zamboni-Rached, M.; Mojahedi, M. Shaping finite-energy diffraction- and attenuation-resistant beams through Bessel-Gauss-beam superposition. Phys. Rev. A 2005, 92, 043839. [CrossRef]

29. Andrews, L.C.; Philips, R.L. Laser Beam Propagation through Random Media; SPIE: Washington, DC, USA, 2005.

30. Nikishov, V.V.; Nikishov, V.I. Spectrum of turbulent fluctuations of the sea-water refraction index. Int. J. Fluid Mech. Res. 2000, 27, 82-98. [CrossRef]

31. Gradshteyn, I.S.; Ryzhik, I.M. Table of Integrals, Series, and Products; Elsevier: Amsterdam, The Netherlands, 2007.

32. Fewell, M.P.; Von, A.; Trojan Absorption of light by water in the region of high transparency: Recommended values for photon-transport calculations. Appl. Opt. 2019, 58, 2408-2421. [CrossRef]

33. Tyler, A.G.; Boyd, R.W. Influence of atmospheric turbulence on the propagation of quantum states of light carrying orbital angular momentum. Opt. Lett. 2009, 34, 142-144. [CrossRef]

(C) 2020 by the authors. Licensee MDPI, Basel, Switzerland. This article is an open access article distributed under the terms and conditions of the Creative Commons Attribution (CC BY) license (http:/ / creativecommons.org/licenses/by/4.0/). 